\title{
A comparison of two survey methods: differences between underwater visual census and baited remote underwater video
}

\author{
Madhavi A. Colton*, Stephen E. Swearer \\ Department of Zoology, University of Melbourne, Parkville, Victoria 3010, Australia
}

\begin{abstract}
Essential to any model, conservation or management plan are measures of the distribution and abundance of a species. Countless methods for estimating these parameters exist, making it essential to assess the limitations and biases associated with a particular sampling protocol. Here, we compare between 2 methods commonly used to survey nearshore fish assemblages. Although most commonly employed, underwater visual census (UVC) may yield biased estimates of abundance depending on the strength of a fish's behavioural response (i.e. avoidance, attraction) to the presence of divers. Baited remote underwater video (BRUV) techniques have shown promise in overcoming some of the limitations of UVC, but are unable to provide an absolute measure of density in turbulent environments. We compare the abilities of these 2 methods to survey the nearshore rocky reef ichthyofauna of Southeast Australia. We found that relative to BRUV, UVC recorded more individuals (in terms of all species, herbivores, cryptic species, and most territorial species), higher richness at both the species and family level, and higher biodiversity as measured using the Shannon Index. These findings remain even when the data were adjusted for differences in sampling effort. In contrast, BRUV recorded proportionally more mobile predators, and a more taxonomically distinct population, though only when taxonomic evenness was not taken into account. Twenty species were unique to UVC and 17 species unique to BRUV. Considering this, studies aimed at cataloguing diversity should apply multiple methods. However, when logistical or financial constraints limit biodiversity studies to only 1 method, UVC will likely provide a more complete estimate of temperate reef fish diversity than BRUV.
\end{abstract}

KEY WORDS: Subtidal fish assemblages $\cdot$ Rocky reefs $\cdot$ Diversity $\cdot$ Herbivores $\cdot$ Territoriality $\cdot$ Mobile predators $\cdot$ Sightability index $\cdot$ Taxonomic distinctness $\cdot$ Victoria $\cdot$ Australia

\section{INTRODUCTION}

Estimates of the abundance and distribution of a species are essential to any ecological model, though measures of these parameters will often be influenced by the method chosen to obtain these data. While the sources of bias within a research protocol may vary, the presence of bias is inevitable (MacNeil et al. 2008a), leaving it to researchers to identify sources of bias within their own data. In the marine environment, accurately assessing population abundance is very challenging. Researchers are limited in the amount of time they can spend monitoring a population and frequently find themselves in conditions that make observations difficult. Considering this, it is unsurprising that numerous techniques have been developed to sample subtidal marine communities, making it essential to compare between methods in order to identify their relative biases.

Bias in surveys can be caused by factors that are intrinsic to the species being observed, as well as by the survey methodology itself. Although most methodologies assume that detectability, or the probability of observing a species, is equal for all species, in reality, 
the fauna of any region is comprised of types of species that are variably detectable (Boulinier et al. 1998, MacNeil et al. 2008a,b). MacNeil et al. (2008a) found that the greatest source of heterogeneity in detectability was caused by species characteristics, i.e. physical traits, behaviour, and life history. To compare between survey methods and identify biases requires classifying the types of species that are differentially detected by each method. In this research, we explore how intrinsic factors influence the detection capacity of 2 methods commonly used to survey subtidal fish assemblages: underwater visual census (UVC) and baited remote underwater video (BRUV).

BRUV has been used to survey species for at least 30 yr (Miller 1975), though its application has increased markedly in recent years, particularly in Australia (e.g. Cappo et al. 2007). BRUV is particularly effective at recording a diverse assemblage of species (Willis \& Babcock 2000, Cappo et al. 2004, Watson et al. 2005). However, BRUV units deployed in turbulent environments are unable to provide measures of absolute density because such measures necessitate that the area surveyed be quantified. This requires detailed information about the dynamics of the bait odour plume, and the sensory capacity, swimming speed, and behaviour of individual species (Priede \& Merrett 1996, Yau et al. 2001, Farnsworth et al. 2007, Heagney et al. 2007), data which are often unavailable for many fishes. The result is that BRUV typically can only provide a measure of relative abundance, particularly in turbulent areas.

In comparison, UVC has a long history of application in surveys of fish assemblages (Brock 1954). The problems associated with the use of UVC to estimate species' densities have been well documented and can be caused by a variety of factors including but not limited to a species' cryptic colouration or secretive behaviour (Sale \& Douglas 1981, Kulbicki 1998), a diver's ability to identify and accurately count individuals (Harvey et al. 2004), and a fish's behavioural response to divers (Kulbicki 1998, Watson \& Harvey 2007). The result of these biases is that UVC usually underestimates fish density (Sale \& Sharp 1983, Edgar et al. 2004).

Few studies have compared UVC to BRUV, and most of these have found BRUV to be superior to UVC at measuring diversity (Willis \& Babcock 2000, Willis et al. 2000, Watson et al. 2005), though at least one study has found the opposite to be true (Stobart et al. 2007). To the best of our knowledge, no studies have examined how these 2 methods compare in measures of taxonomic diversity. If BRUV is better at recording mobile predators (Willis \& Babcock 2000, Cappo et al. 2004, Watson et al. 2005, Watson \& Harvey 2007), including many elasmobranchs, then it may survey a more diverse assemblage. If, however, UVC is equally good at surveying these species (Friedlander \& DeMartini 2002, Castro \& Rosa 2005, Powter \& Gladstone 2008) then there may be little difference between the methods in measures of taxonomic distinctness. In this research, we were interested not only in how these 2 methods differ in various measures of diversity and richness, but also in how factors that are intrinsic to a species may influence how well a method detects a species. Specifically we were interested in the effects of conspicuousness, crypsis, mobility, territoriality, trophic position and schooling behaviour on detectability using BRUV and UVC.

Conspicuous species are perhaps the easiest to survey, with numerous methods developed to measure their densities. However, being conspicuous alone is not enough to guarantee observation. For example, MacNeil et al. (2008a) found that scarids had a relatively low probability of detection despite their physical conspicuousness, and attributed this incongruity to the species' highly mobile nature and diver-averse behaviour. The behaviour of many fishes changes in the presence of divers (Kulbicki 1998, Watson \& Harvey 2007), which may explain why some researchers have found BRUV to be especially effective at observing predatory and mobile species, such as elasmobranchs or species that are targeted by fishing (Willis \& Babcock 2000, Cappo et al. 2004, Watson et al. 2005, Watson \& Harvey 2007). Other research, however, demonstrates that mobile predators can be accurately surveyed using UVC (e.g. Friedlander \& DeMartini 2002, Castro \& Rosa 2005, Robbins et al. 2006). In our research, we investigate differences in how the 2 methods measure abundance and richness of both conspicuous species and mobile predators.

In the detection spectrum, cryptic species are at the opposite end to conspicuous species. These fishes are rarely detected in visual surveys, which are well known to underestimate their density (Willis 2001). In fact, the only way to accurately survey cryptics may be with the application of an ichthyocide (Willis 2001). While the chance of detecting cryptic species is low on a UVC, it is even smaller when using diver-swum underwater video (Watson et al. 2005). When the underwater video is stationary, as in the case of a BRUV, the chance of seeing cryptic species declines further (Stobart et al. 2007). In addition to cryptic colouration and secretive behaviour, cryptic species often have small maximum total lengths ( $\mathrm{TL}_{\mathrm{max}}$ ). Interestingly, MacNeil et al. (2008b) found $\mathrm{TL}_{\max }$ to be negatively associated with detection probability, and proposed that the territorial behaviour of the small-bodied species in their study compensated for their small size, with the result that these small-bodied species were more detectable than larger-bodied transitory species. The effect that territoriality has upon survey methodol- 
ogy has received little attention in the literature. There are good a priori reasons to expect that UVC and BRUV might provide different estimates of the abundance of territorial species. A diver conducting a UVC is likely to pass through many individuals' territories, while a BRUV unit will usually land in a single individual's territory or the junction area between a few individuals' territories. Similar to Willis \& Babcock (2000), we frequently observed individuals interacting in an antagonistic manner around the bait. We predicted that the result of these behaviours would limit a territorial species' ability to respond to the bait, thereby allowing UVC to record higher abundances of these species than BRUV.

Another group of species that might be limited in their response to bait are herbivores. While it is tempting to suppose that BRUV does not survey herbivores at all, this is not the case. Harvey et al. (2007) found that herbivores were attracted to a BRUV more than to an unbaited video unit, with attraction most likely being to the activity around the frame rather than to the bait itself. However, this study did not compare herbivore densities measured using BRUV to those measured using UVC. In our research, we explore differences in abundances measured using UVC and BRUV with the specific prediction that UVC will record higher abundance of herbivores.

One of the identified sources of bias using UVC is the difficulty that divers may have in accurately counting individuals (Harvey et al. 2004), which could be especially true if a species forms dense schools. While schooling behaviour increases the chance that a species will be detected (MacNeil et al. 2008b), it is unclear whether UVC can provide an accurate estimate of this group's abundance. Measures of abundance of these species using BRUV may also be compromised: There is an upper limit to the number of fish that can be viewed in a frame (Willis et al. 2000). Here, we explore differences in these methods' abilities to record abundance and richness of densely schooling species.
Our methodological comparison utilizes the diverse fish assemblages inhabiting temperate rocky reefs in Southeast Australia. This assemblage has received little scientific investigation and there is a pressing need to establish appropriate survey protocols for (1) assessing the response of this assemblage to marine protected area (MPA) establishment, (2) identifying locations/species of high conservation value, and (3) testing the applicability of the bioregional province framework developed for the management of southern Australian marine waters to reef fishes. We utilize this assemblage to compare performance of these 2 methods in quantifying diversity as well as to explore intrinsic factors that influence a method's ability to detect a species. No comparison between methods is complete without an assessment of effort. While some research has found BRUV stations to be more efficient in terms of statistical power, personnel hours, and boat resources (Cappo et al. 2003, Watson et al. 2005), each research program will differ in resource availability and application. Here, we compute the amount of time to conduct a single sample of each method in order to compare performance of both methods using a standardized metric.

\section{MATERIALS AND METHODS}

Locations. Surveys were conducted on nearshore rocky reefs at 4 locations along the coast of Victoria in Southeast Australia (Fig. 1) between December 2007 and June 2008 (Table 1). Locations were chosen to include MPAs, and to be representative of 3 biogeographic provinces and 1 transition zone (Hough \& Mahon 1994). To ensure that surveys occurred in the correct habitat in a patchy matrix of sand and rocky reef, we selected sites based on depth (between 4 and $20 \mathrm{~m}$ ) and real-time side scan sonar imagery of the benthos. We aimed to conduct 1 UVC for every 3 BRUV units deployed. However, due to a combination of equipment malfunction and poor weather, we were

Table 1. Sampling effort by method at each location. Start and end dates and number of days spent sampling for both methods combined. Median number of days that separated the application of the 2 methods is provided for each location. Distance and depth: $(\mathrm{m})$, times: $(\mathrm{min})$, swim speed: $\left(\mathrm{m} \mathrm{min}^{-1}\right)$. For BRUV, $\mathrm{T}_{\text {soak }}=$ total time the frame is immersed, and $\mathrm{T}_{\text {depth }}=$ time the frame spends at depth. All values are mean $\pm \mathrm{SD}$

\begin{tabular}{|c|c|c|c|c|c|c|c|c|c|c|c|}
\hline \multirow{2}{*}{ Location } & \multirow{2}{*}{ Start date } & \multicolumn{3}{|c|}{ UVC \& BRUV } & \multirow[b]{2}{*}{ Depth } & \multirow[b]{2}{*}{ Distance } & \multicolumn{2}{|l|}{$-\mathrm{UVC}_{-}$} & \multirow[b]{2}{*}{ Depth } & \multirow{2}{*}{$\begin{array}{c}-\mathrm{BRUV}- \\
\mathrm{T}_{\mathrm{soak}}\end{array}$} & \multirow[b]{2}{*}{$\mathrm{T}_{\text {depth }}$} \\
\hline & & End date & No. days & $\begin{array}{c}\text { Median no. } \\
\text { days b/w } \\
\text { methods }\end{array}$ & & & Speed & Duration & & & \\
\hline Apollo & 22 Jan 08 & 03 Mar 08 & 41 & 34 & $13.5 \pm 4.8$ & $502 \pm 300$ & $14 \pm 9$ & $34 \pm 8$ & $11.2 \pm 5.0$ & $59.8 \pm 4.0$ & $60.0 \pm 2.0$ \\
\hline Barwon & 19 Dec 07 & 17 Jun 08 & 181 & 155 & $10.6 \pm 4.5$ & $261 \pm 84$ & $10 \pm 2$ & $27 \pm 7$ & $10.3 \pm 4.7$ & $61.0 \pm 2.7$ & $59.2 \pm 4.7$ \\
\hline Prom & 18 Mar 08 & 05 Jun 08 & 79 & 70 & $12.6 \pm 4.7$ & $272 \pm 70$ & $9 \pm 3$ & $30 \pm 1$ & $11.7 \pm 4.7$ & $61.8 \pm 0.4$ & $61.2 \pm 0.5$ \\
\hline Howe & 05 Apr 08 & 19 Apr 08 & 14 & 10 & $14.1 \pm 3.9$ & $314 \pm 102$ & $9 \pm 4$ & $37 \pm 7$ & $13.6 \pm 4.2$ & $58.3 \pm 8.8$ & $56.7 \pm 9.0$ \\
\hline
\end{tabular}


Fig. 1. Number of surveys, n, conducted using each method at locations along the coast of Victoria in Southeast Australia. An inset of one location, Wilsons Promontory, is provided to illustrate the positioning of replicate BRUV deployments relative to the paired UVC; $\boldsymbol{\square}=$ BRUV replicates, $\Delta=$ start of UVC transects, $\Delta=$ end of UVC transects
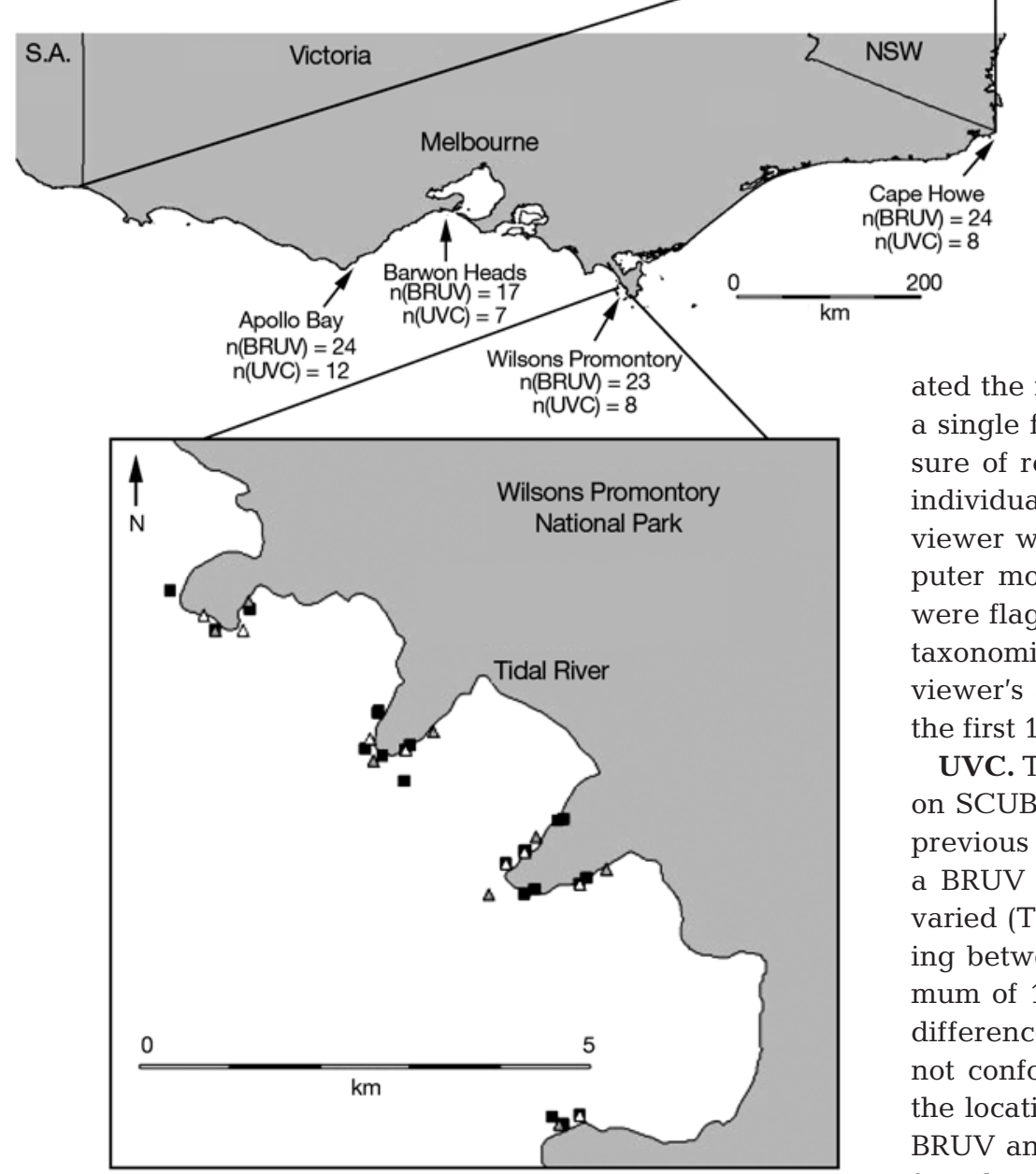

unable to achieve this ratio at all sites. The actual numbers of surveys of each type are provided in Fig. 1.

BRUV. To capture underwater video footage, we placed an HC-series Sony Handycam in an Ocean Images ${ }^{\mathrm{TM}}$ underwater housing that was mounted into a weighted aluminium frame (Cappo 2006). A tube of PVC extended from the frame's lid, from which we suspended a mesh bait pouch containing $400 \mathrm{~g}$ of crushed pilchards, $1.5 \mathrm{~m}$ from the lens (Fig. 2). Trial deployments with the bait arm attached beneath the camera were unsuccessful as kelp often occluded the bait bag. The bait pouch rested within $\sim 20 \mathrm{~cm}$ of the benthos when full and $\sim 30 \mathrm{~cm}$ above when empty. The unit was deployed from a boat onto or immediately next to rocky reef. After deployment, the boat motored away from the area. We deployed 2 BRUV units concurrently at a minimum of $500 \mathrm{~m}$ apart to minimize the overlap of bait odour plumes (Willis \& Babcock 2000, Harvey et al. 2007, Heagney et al. 2007). Where BRUV units could not be separated spatially, replicates occurring $<500 \mathrm{~m}$ apart were deployed at least $1.5 \mathrm{~h}$ apart.

Preliminary data collected from Barwon Heads suggested that BRUV immersions of 60 min duration were required to record many species in Victorian coastal waters, as $47 \%$ of maximum species counts occurred after $30 \mathrm{~min}$, and $24 \%$ occurred after $45 \mathrm{~min}$ (M. A. Colton unpubl. data). For each species on each tape, we enumerated the maximum number of individuals observed in a single frame $(\operatorname{Max} N) . \operatorname{Max} N$ is a conservative measure of relative density that avoids the recounting of individuals that repeatedly visit the bait. A single viewer watched all the tapes at least once on a computer monitor. Species of questionable identification were flagged and viewed again, and the assistance of taxonomists ${ }^{1}$ was called upon when necessary. As the viewer's ability to identify fishes improved over time, the first 12 tapes examined were viewed twice.

UVC. To conduct UVCs, an observer and dive buddy on SCUBA descended to the substrate at the site of a previous BRUV deployment (Fig. 1). The time between a BRUV deployment and a subsequent UVC survey varied (Table 1), with a median number of 39 d elapsing between survey types at all locations and a minimum of $1 \mathrm{~d}$ at any location. We tested to ensure that differences in the time elapsed between methods did not confound our results by removing from analyses the location with the largest number of days between BRUV and UVC surveys (below). After waiting 2 min for the disturbance of their arrival to dissipate (Samoilys \& Carlos 2000), the observer swam slowly in a pre-determined direction, looking ahead as well as under ledges and in the kelp understory, identifying and counting fish in a strip $5 \mathrm{~m}$ wide. The dive buddy, present for safety reasons, remained behind the observer. The direction of each census was chosen prior to the dive and was based upon the strength and direction of currents, as well as the ability of the boat to retrieve the divers. The latitude and longitude of diver entry and egress points were used to compute the lin-

${ }^{1}$ Dr. Martin F. Gomon, Senior Curator, and Dianne J. Bray, Fish Collections Manager, Museum Victoria, Carlton, Victoria 3053, Australia 


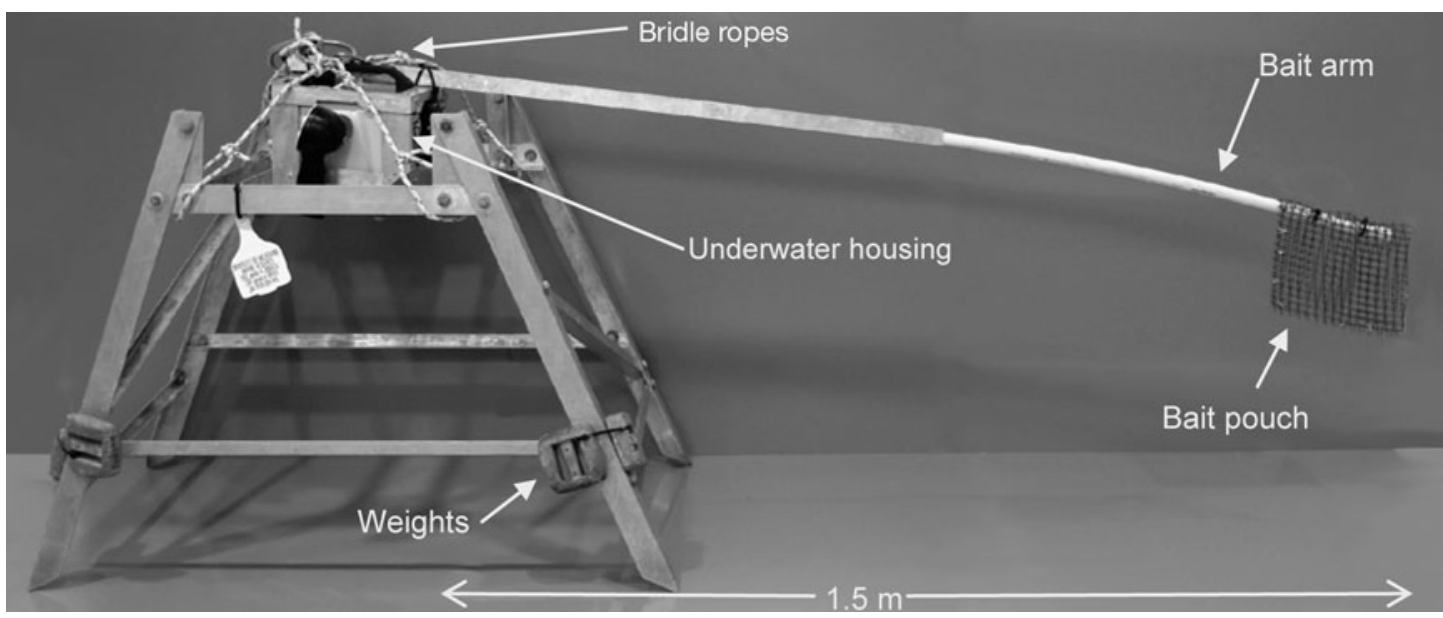

Fig. 2. BRUV unit. Frame constructed of aluminium; bait arm is PVC pipe; bait pouch is gutter mesh; bridle ropes connect to a surface buoy and allow the unit to be deployed and retrieved remotely; 2 lead dive weights (1.5 kg each) attached to each leg; video camera mounted into underwater housing

ear transect distance. Though divers made every effort to swim in a straight line using a compass heading, deviations were inevitable, making the linear survey distance an underestimate of the actual area surveyed. However, deviations from the straight line distance will represent only a small proportion of the total survey area because transects were so long (Table 1). The dive time was limited by no-decompression limits in dive tables or by tank duration (Table 1). Swimming speeds were comparable to those in other surveys (see Lincoln Smith 1988 for a review) (Table 1). All observers were highly trained in fish identification, and $80 \%$ of UVCs were conducted by the same researcher.

Lincoln Smith (1988) found that differences in observer responses to either time-based or distancebased methods can result in biased estimates of some species' abundances. We chose to conduct UVCs with unregulated distances and times, allowing habitat complexity, the presence of fish, and currents to determine our swimming speed. Like Fowler (1987), we found no relationship between $\log _{\mathrm{e}}$-transformed transect length and species richness, diversity, or number of individuals for all locations combined using Pearson product-moment correlations $(\mathrm{r}=-0.05, \mathrm{r}=0.19, \mathrm{r}=$ -0.06 respectively; all $n=35, p>0.05$ ) (M. A. Colton unpubl. data). We avoided the use of transect tape in order to more effectively use our bottom time (Patterson et al. 2007), reduce our disturbance (Fowler 1987), and conduct long-distance UVCs without running into problems arising from the deployment of long lengths of tape in considerable surge. Unfortunately, the use of sonar to measure transect lengths (Patterson et al. 2007) was not found effective in the turbid waters which typify our study sites.
Between-methods comparisons using relative abundance. We examined differences in species composition using relative abundance, measured as relative density using UVC and relative $\operatorname{MaxN}$ using BRUV. For univariate analyses in which the information in a sample was condensed to a single number, e.g. Shannon Diversity, we computed relative abundance as the total number of individuals in a sample divided by the total number of individuals observed in all samples using that method. For multivariate analyses, we computed the relative abundance of a species in a sample by dividing the number of individuals of a species in a sample by the total number of individuals in the sample. Means were computed using all samples only from the location(s) in which a species was observed. Individuals were identified to species wherever possible. There were, however, some differences in the ability of the methods to identify fishes: using BRUV, it was difficult to differentiate between species in the families Diodontidae and Urolophidae, though these could be distinguished using UVC. For comparisons between methods, species observed using UVC from these 2 families were combined into 2 familylevel groupings.

Diversity and abundance. Measures of diversity were compared between the methods using univariate analyses, and mean relative abundances were compared using multivariate analyses. As UVCs were conducted at approximately the same sites in which BRUVs were deployed, we used paired $t$-tests (SPSS v16.0) to compare measures of diversity, which controlled for differences in habitat that naturally occur in patchy reef environments. Before conducting $t$-tests, all dependent variables were tested for normality, and a fourth-root transformation was applied to normalize 
the variances of number of individuals as the data were highly skewed. As mentioned previously, we attempted to always compare 3 BRUV deployments with $1 \mathrm{UVC}$, though technical difficulties occasionally precluded achievement of this ratio. Therefore, mean values computed from up to 3 BRUV deployments were compared with the value computed from 1 UVC. We examined species richness, family richness, fourth-root transformed number of individuals and diversity as measured by the Shannon Index:

$$
H^{\prime}=\sum_{i=1}^{s}\left(p_{i}\right) \ln \left(p_{i}\right)
$$

where $p_{i}$ is the proportion of individuals in a sample belonging to species $i$.

Differences in diversity between the methods were also examined using a multivariate approach. We employed the ANOSIM routine in Primer-E (Clarke \& Warwick 2001a) to compare between methods including the factor location, using a Bray-Curtis dissimilarity matrix of fourth-root transformed relative abundance. Those species responsible for the observed differences were identified using the SIMPER routine.

As UVCs and BRUVs were conducted within the same season at all locations except Barwon Heads (Table 1), we repeated our univariate and multivariate analyses omitting Barwon Heads, and assessed significance using a Bonferroni adjusted $\alpha$-level of 0.03 .

Taxonomic distinctness. In addition to species richness and diversity, we tested for between-methods differences in taxonomic distinctness. A Linnaean classification system was used including the ranks species, genus, sub-family, family, order, and class. Assignments to species, genus, sub-family and family were based on the Catalog of Fishes (Eschmeyer \& Fricke 2009), while the ranks of order and class were obtained from Gomon et al. (2008). Two measures of taxonomic diversity were examined: $\Delta^{+}$, or the average path length between species (Clarke \& Warwick 1998); and $\Lambda^{+}$, or the variance of the pairwise path lengths (Clarke \& Warwick 2001b). We used the TAXDIST routine in PRIMER-E (Clarke \& Warwick 2001a) to create funnel plots with $95 \%$ confidence intervals for presence/ absence data collected using both methods. Equal weights were assigned to all taxonomic ranks; changing the weight for sub-family to half that of the other ranks was found to make no difference to the results.

Intrinsic factors affecting detectability. In addition to measures of diversity and richness, we also examined which kinds of species were best recorded by each method. Species were classified into sightability types using an adaptation of the criteria developed by Lincoln Smith (1989). Type I species were classified as site-attached, often small-bodied or cryptic, e.g. Parma microlepis (Pomacentridae). Type II species form dense schools in the water column, e.g. Atypichthys strigatus (Microcanthinae). Type III species are conspicuous, either occurring as individuals or in small groups, e.g. Notolabrus tetricus (Labridae).

In addition to sightability, we tested whether territorial species, herbivores, or mobile predators were better sampled by either method. We hypothesized that herbivorous species would not be attracted to the bait in as high numbers as carnivorous or omnivorous species, and that higher abundances of territorial species would be recorded by UVC as these species could be limited in their ability to respond to bait. We also predicted that large, mobile predators, such as many elasmobranchs, would be better sampled by BRUV than UVC, as has been shown by some other studies (Willis \& Babcock 2000, Cappo et al. 2004, Watson et al. 2005, Watson \& Harvey 2007). We identified species belonging to the 3 groups using data from guidebooks (Kuiter 2000, Edgar 2005, Gomon et al. 2008). We used independent samples $t$-tests, with degrees of freedom computed using the Welch-Satterthwaite formula in SPSS (v 16.0) to correct for unequal sample sizes and heteroscedasticity, to examine whether the methods differed in the mean number of species $([\ln (x+1)]$-transformed) of each sightability type. We also tested whether the relative abundance (fourth-root transformed) of species of the different sightability types and groups (herbivores, territorial species, and mobile predators) differed between methods. We used a Bonferoni-adjusted $\alpha$-level of 0.03 to assess significance.

Between methods comparison of effort. We compared effort between methods firstly by using species accumulation curves to estimate the maximum species richness for each method, and secondly by computing the number of samples required by each method to observe a proportion of the maximum species richness. Finally, we computed a metric that standardized these samples, allowing us to compute the amount of effort, measured in time, required by each method to achieve a proportion of maximum species richness.

Species accumulation curves were constructed using EstimateS (v 8.00, Colwell 2006) for UVC and BRUV separately at each location. Species accumulation curves assume that replicate samples are equal. Although oceanographic conditions, bait dispersal and therefore the area surveyed by a BRUV unit will not always be the same among deployments, and individual UVC transects also sampled different areas (Table 1), we make the simplifying assumption that BRUV deployments are equal and that one UVC sample is equivalent to the mean distance covered in all transects (mean $\pm \mathrm{SD}=360 \pm 212 \mathrm{~m}$ ). However, as EstimateS randomises the ordering of samples during the simulations, such potential variation among sam- 
ples will simply widen the confidence intervals of the estimate.

To estimate the maximum species richness observable for each method, we used NLREG (Sherrod 2008) to fit the Michaelis-Menten function (Raaijmakers 1987 ) using $S_{\text {obs }}$ (Mao $\tau$ ), the observed species richness calculated by EstimateS, as a measure of $S(x)$, which is the number of species, $S$, observed at a given level of sampling effort, $x$ :

$$
S(x)=\frac{x\left(S_{\max }\right)}{b+x}
$$

where $b$ and $S_{\max }$ are constants, and $S_{\max }$ is also the predicted asymptotic species richness. We assessed goodness of fit using $\mathrm{r}^{2}$ values calculated by NLREG, all of which were $>0.95$.

To determine the number of samples required to obtain a certain percentage of predicted species richness, we solved Eq. (2) for $x$ given $y=Z\left(S_{\max }\right)$, where $Z$ is a proportion of the predicted species richness $\left(S_{\max }\right)$ :

$$
x=\frac{-Z b}{Z-1}
$$

We then computed $x$ given $Z=60 \%, 75 \%$, and $95 \%$ for each method and location.

Finally, we determined how much time it took to complete a single sample using each method, and utilized these values to compute a standardization metric. To complete a sample, each method requires time in the field and in the lab, and, for our research program, the methods required the same number of personnel in both settings. As the parameters required to convert time to cost will be specific to a research program, here we only report the time taken in our surveys to deploy a single BRUV unit and conduct 1 UVC transect. Our research used 2 BRUV units, which we deployed from a small vessel (6 m length).

We computed the mean field-time to deploy a single BRUV unit from 14 sampling days in which we only conducted BRUV surveys. To each day of sampling, we added 20 min per camera to account for the time to set up and break down the equipment. The mean time to launch and retrieve a single BRUV unit, including time spent recording, was 54 field-min. There is a wellknown bottleneck (Cappo et al. 2003) in the analysis of BRUV tapes, with the time to analyze a tape dependent upon the number of individuals on the tape and the ease by which species can be identified. Estimates of time spent in the lab on BRUV analysis range from $2 \times$ the length of the tape (Willis et al. 2000) to $24 \times$ the time spent on a UVC (Stobart et al. 2007). In our computations, we use an estimate of $2 \times$ BRUV tape length as a measure of minimum analysis time. In addition, we speculate that it takes $\sim 20$ min to enter the data collected from the tape into an appropriate computer pro- gram. Therefore, the total mean time to deploy, retrieve and analyze a single BRUV tape is 190 min.

To compute the time spent conducting a single UVC, we took the mean survey time plus 15 min spent gearing up at the start of a dive, 8 additional min in the water ( 1 min to reach the benthos, a 2 min wait period at the start of the dive, 2 min to the surface, and $3 \mathrm{~min}$ on a safety stop), and 5 min getting onto the boat at the end of a dive. Lab time for UVC is minimal: we speculated that it takes $\sim 20 \mathrm{~min}$ to enter the data into an appropriate computer program. This resulted in an average of $~ 80 \mathrm{~min}$ for 1 UVC. Using these times, we computed that we could complete about 2 UVC transects for every 1 BRUV unit deployed. To determine the amount of time required to obtain the same percentages of total species richness for each method, we multiplied the number of BRUV samples by 2 to standardize for the between-method differences in the time taken to obtain a sample.

\section{RESULTS}

We observed a total of 78 species belonging to 44 families using BRUV, and 85 species belonging to 42 families using UVC (Fig. 3). Seventeen species were only observed by BRUV, and 20 species were only observed by UVC. Of those species that were frequently observed, i.e. within the upper 50th percentile, only 3 were exclusively observed using UVC and only 2 were exclusively observed using BRUV (Fig. 3). The 3 families occurring most frequently in BRUV samples were Labridae (observed in $89 \%$ of the samples), Monacanthidae (66\%), and Carangidae (58\%). The 3 families that occurred most frequently in UVC samples were Labridae (100\% of samples), Monacanthidae (100\%), and Cheilodactylidae (89\%).

\section{Diversity and abundance}

Using paired $t$-tests, we found that UVC recorded significantly higher species diversity $\left(t_{(34)}=3.66, \mathrm{p}=0.001\right)$, species richness $\left(t_{(34)}=7.19, \mathrm{p}<0.0005\right)$, family richness $\left(t_{(34)}=6.30, \mathrm{p}<0.0005\right)$, and number of individuals $\left(t_{(34)}=\right.$ 10.94, $\mathrm{p}<0.0005)$ than BRUV when all locations were examined (Fig. 4) and when the Barwon Heads site was excluded from the analysis (Shannon Index diversity $t_{(27)}=3.11, \mathrm{p}=0.004 ;$ species richness $t_{(27)}=4.33, \mathrm{p}<$ 0.0005 ; family richness $t_{(27)}=3.8, \mathrm{p}=0.001$; and number of individuals $\left.t_{(27)}=11.551, \mathrm{p}<0.0005\right)$.

Using the ANOSIM routine in Primer-E (Clarke \& Warwick 2001a), we found a significant difference between methods in the relative abundance of species for all locations $(\mathrm{R}=0.225, \mathrm{p}<0.001)$ (Fig. 5), and when 

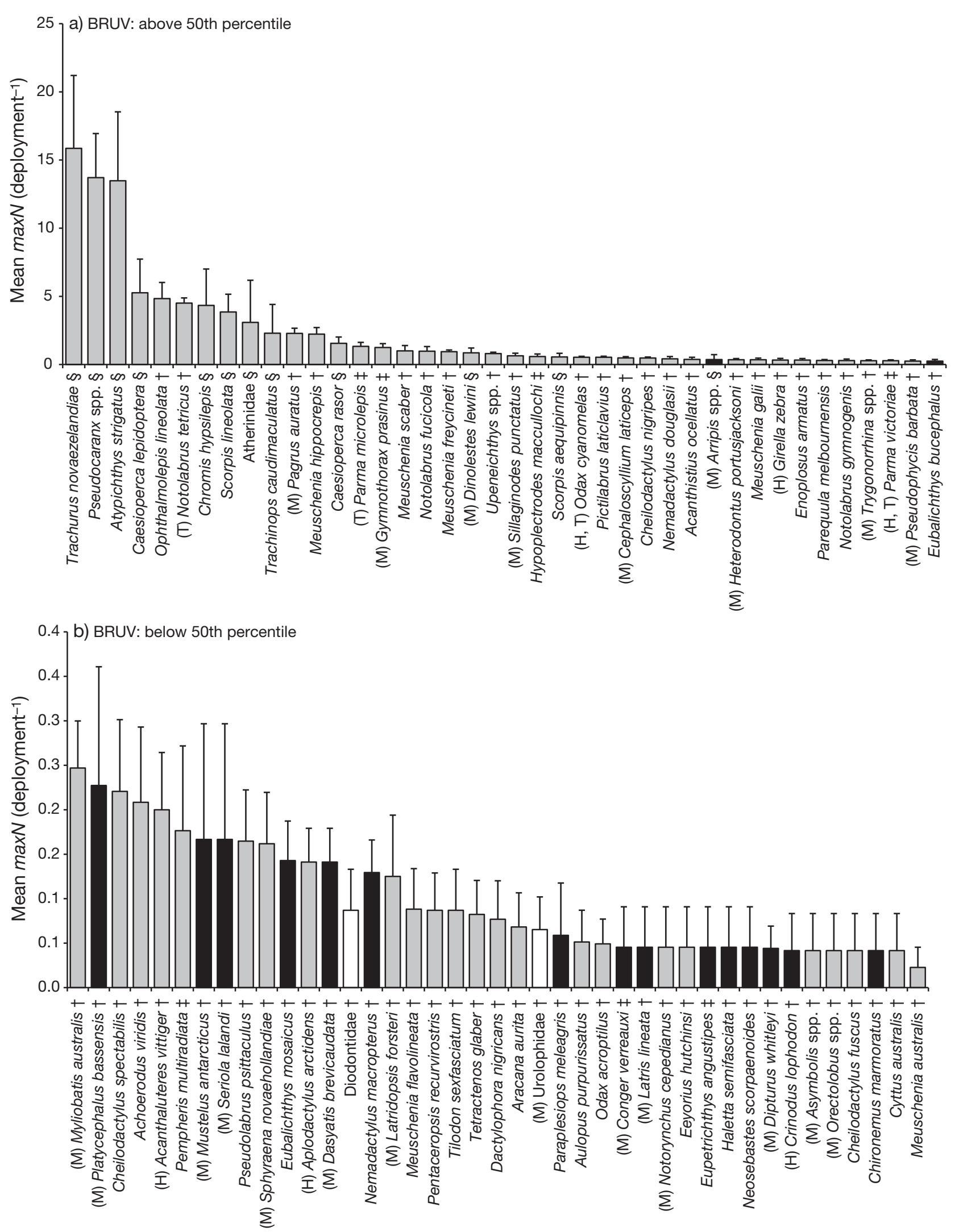

Fig. 3. (Above and facing page.) Mean abundance measured by (a) \& (b) mean $\max N$ for BRUV samples, and (c) \& (d) mean density $\left(\mathrm{m}^{-2}\right)$ for UVC samples. Species shown in (a) \& (c) are those above the 50th percentile of abundance, and (b) \& (d) are those below the 50th percentile of abundance. Means were computed only from location(s) in which species occurred. Sightability types (see 'Materials and Methods') are shown after species' names, with: $\ddagger=$ Type I, $\S=$ Type II, $\dagger=$ Type III. Letters in parentheses at the start of a species' name indicate group, with: $H=$ herbivores, $T=$ territorial species, $M=$ mobile predators. Black bars $=$ species unique to a single method; white bars = species from families that could be identified to species level using UVC but not BRUV; grey bars = species that could be identified to species level and that were observed by both methods. Error bars are + SE 

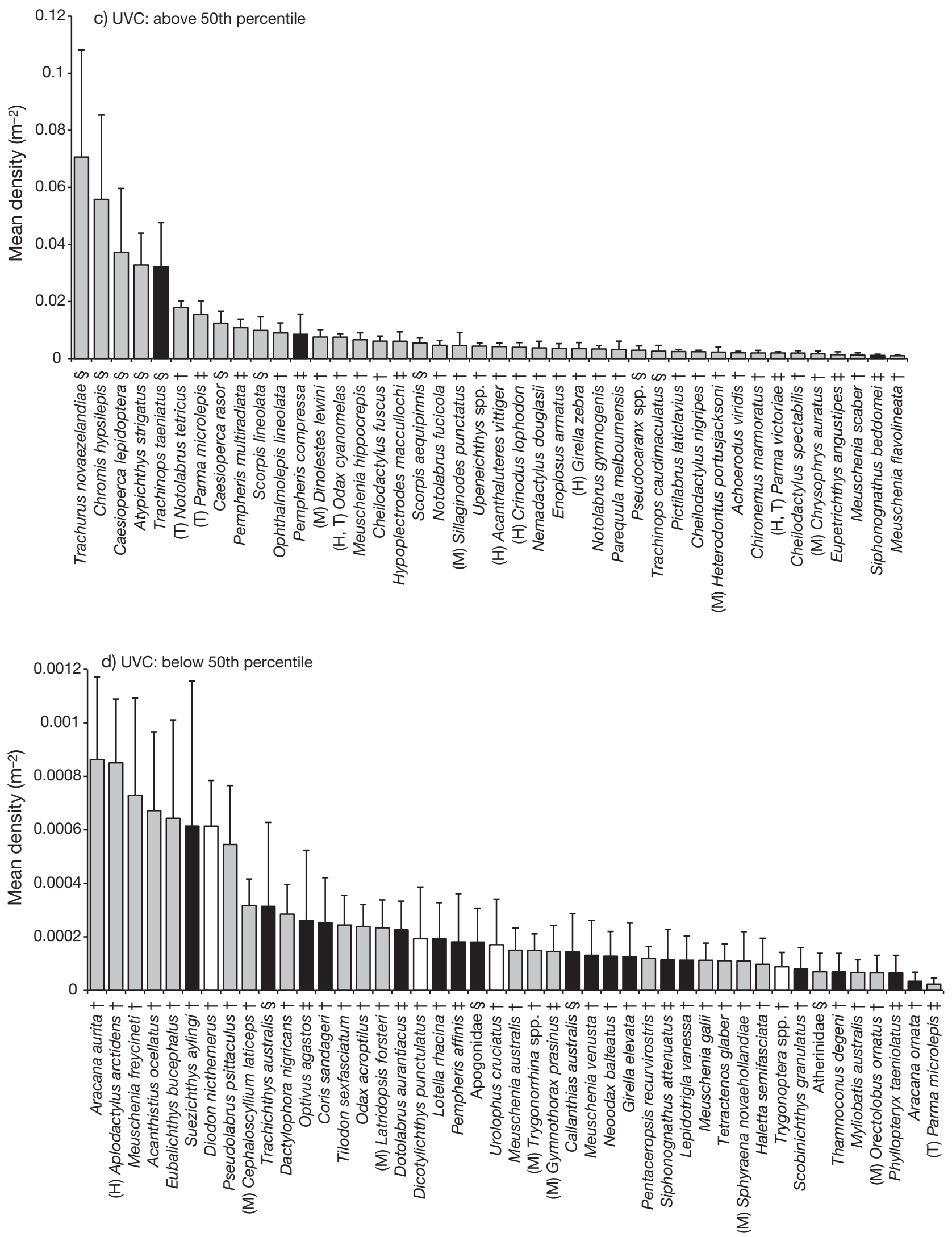

Fig. 3 (continued) 


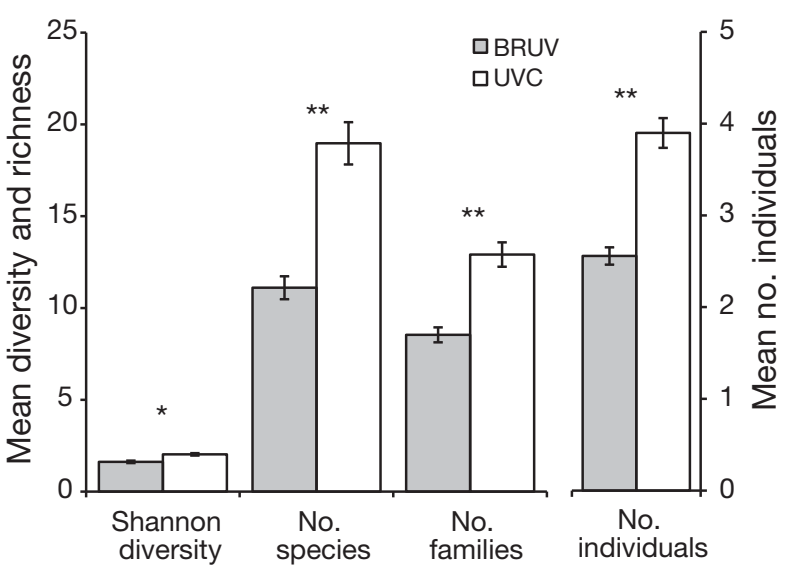

Fig. 4. Paired $t$-tests comparing diversity and abundance between BRUV and UVC for all locations. Asterisks indicate significance: ${ }^{*} \mathrm{p}=0.001 ;{ }^{* *} \mathrm{p}<0.0005$. Error bars are $\pm \mathrm{SE}$

Barwon Heads was excluded $(\mathrm{R}=0.218, \mathrm{p}<0.004)$. Using the SIMPER routine, we found that 16 species were required to explain $>50 \%$ of the difference between methods, and that no single species contributed $>4.5 \%$ (Table 2 ). Notably, the average abundances of the top 4 species differed between BRUV and UVC, with half observed in higher numbers by BRUV, and half by UVC.

\section{Taxonomic distinctness}

We examined how the methods compared in 2 measures of taxonomic distinctness, $\Delta^{+}$and $\Lambda^{+}$, using the TAXDIST routine in Primer-E (Clarke \& Warwick 2001a). UVC was found to survey a less taxonomically distinct population, i.e. to have lower $\Delta^{+}$, than BRUV (Fig. 6a). However, when the evenness of the taxonomic tree, i.e. $\Lambda^{+}$, was taken into account, there was little difference between methods (Fig. 6b).

\section{Intrinsic factors affecting detectability}

Both methods were good at detecting conspicuous (Type III) species and poor at detecting cryptic (Type I) species. Of all the species observed, $73 \%$ were classified as Type III, $15 \%$ as Type I and $12 \%$ as Type II

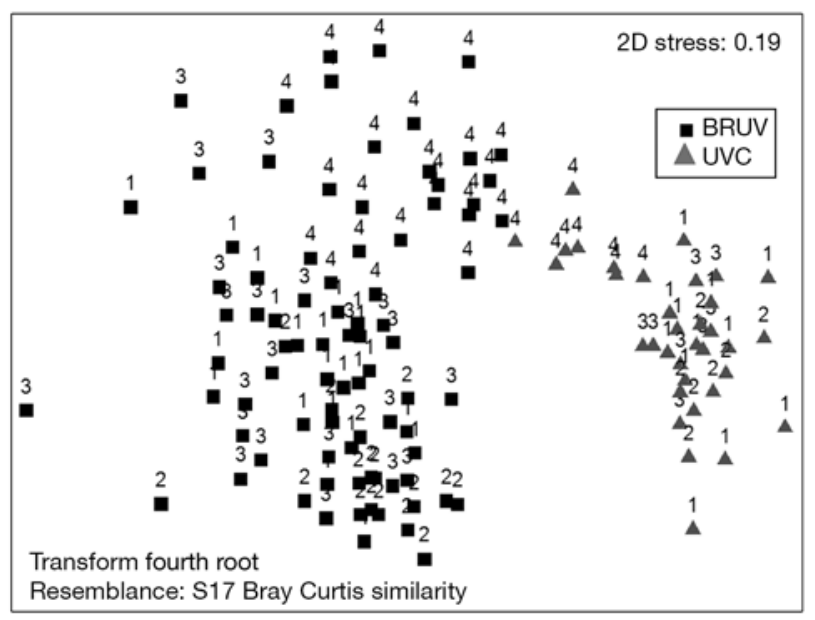

Fig. 5. MDS plot of ANOSIM testing differences between methods including factor location. Numbers represent locations: 1 = Apollo Bay, 2 = Barwon Heads, 3 = Wilsons Promontory, and $4=$ Cape Howe

Table 2. Results from SIMPER routine in Primer-E used to identify species contributing to differences between methods. Species are listed in order of their contribution. Mean relative abundances, $\bar{X} \pm \mathrm{SE}$, listed for each method and computed from only the location(s) in which a species was observed

\begin{tabular}{|c|c|c|c|c|c|}
\hline Species & Family & $\bar{X}_{(\mathrm{UVC})}$ & $\bar{X}_{\text {(BRUV) }}$ & $\%$ contribution & Cumulative \% \\
\hline Chrysophrys auratus & Sparidae & $0.004 \pm 0.002$ & $0.064 \pm 0.013$ & 4.5 & 4.5 \\
\hline Odax cyanomelas & Odacidae & $0.051 \pm 0.008$ & $0.017 \pm 0.003$ & 4.0 & 8.5 \\
\hline Pseudocaranx spp. & Carangidae & $0.014 \pm 0.011$ & $0.180 \pm 0.030$ & 3.8 & 12.3 \\
\hline Pempheris multiradiata & Pempheridae & $0.052 \pm 0.017$ & $0.005 \pm 0.004$ & 3.6 & 15.9 \\
\hline Upeneichthys spp. & Mullidae & $0.027 \pm 0.006$ & $0.032 \pm 0.007$ & 3.5 & 19.4 \\
\hline Notolabrus fucicola & Labridae & $0.033 \pm 0.012$ & $0.023 \pm 0.008$ & 3.3 & 22.8 \\
\hline Acanthaluteres vittiger & Monacanthidae & $0.029 \pm 0.006$ & $0.005 \pm 0.002$ & 3.3 & 26.0 \\
\hline Meuschenia freycineti & Monacanthidae & $0.008 \pm 0.004$ & $0.022 \pm 0.004$ & 3.2 & 29.2 \\
\hline Caesioperca rasor & Serranidae & $0.084 \pm 0.030$ & $0.039 \pm 0.011$ & 3.1 & 32.3 \\
\hline Pictilabrus laticlavius & Labridae & $0.015 \pm 0.003$ & $0.018 \pm 0.004$ & 3.1 & 35.3 \\
\hline Meuschenia hippocrepis & Monacanthidae & $0.077 \pm 0.030$ & $0.063 \pm 0.009$ & 3.0 & 38.3 \\
\hline Cheilodactylus nigripes & Cheilodactylidae & $0.028 \pm 0.006$ & $0.021 \pm 0.004$ & 3.0 & 41.3 \\
\hline Dinolestes lewini & Dinolestidae & $0.054 \pm 0.020$ & $0.017 \pm 0.006$ & 2.9 & 44.3 \\
\hline Enoplosus armatus & Enoplosidae & $0.017 \pm 0.006$ & $0.011 \pm 0.005$ & 2.9 & 47.2 \\
\hline Scorpis aequipinnis & Kyphosidae & $0.045 \pm 0.013$ & $0.013 \pm 0.005$ & 2.8 & 49.9 \\
\hline Girella zebra & Kyphosidae & $0.028 \pm 0.017$ & $0.009 \pm 0.004$ & 2.7 & 52.6 \\
\hline
\end{tabular}



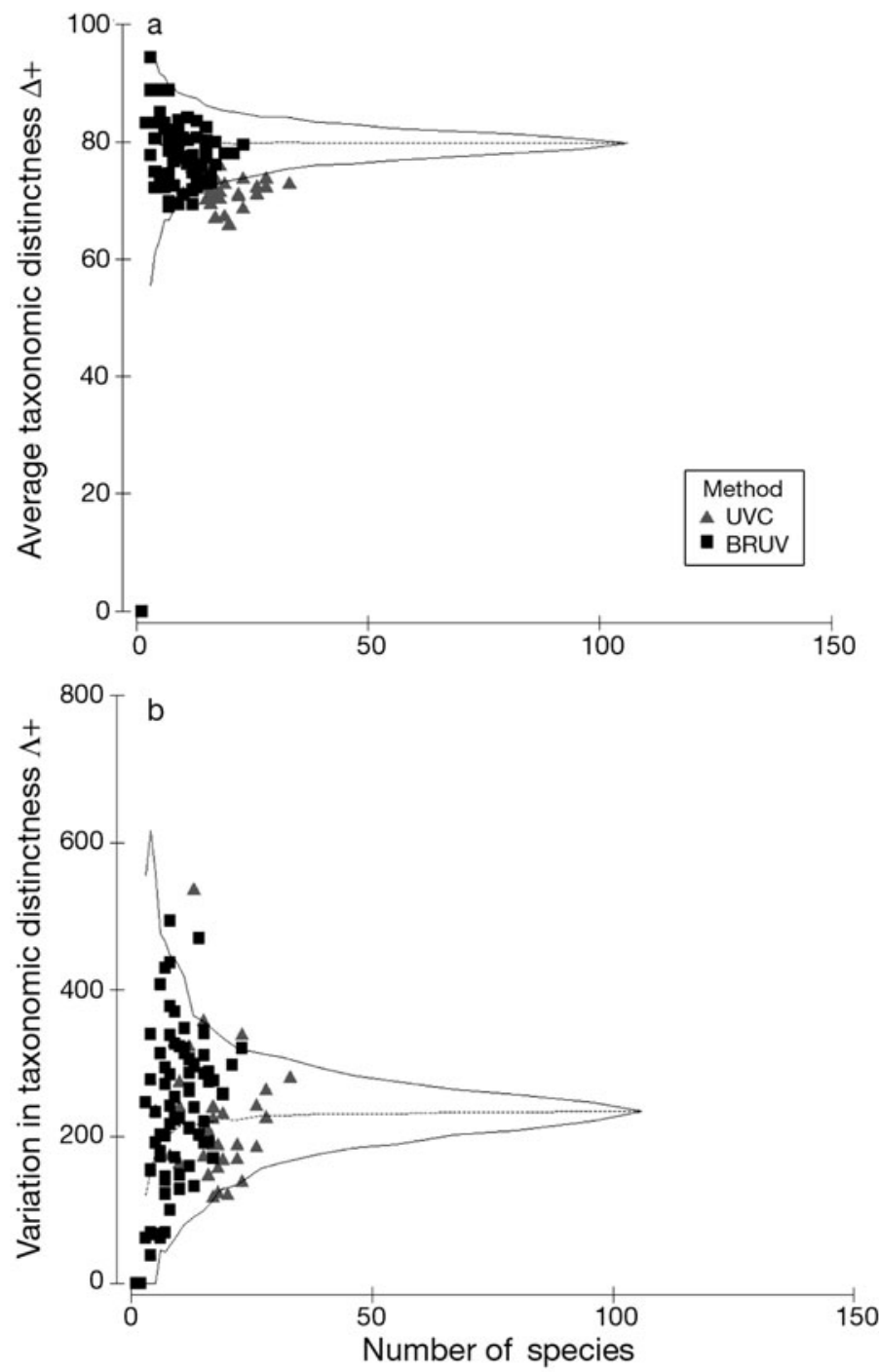

Fig. 6. Differences between methods in 2 measures of taxonomic diversity: (a) taxonomic distinctness $\Delta^{+}$, and (b) variation in taxonomic distinctness $\Lambda^{+}$. Solid lines $=95 \%$ confidence intervals; dashed lines $=$ mean

(Fig. 3). Of those species observed only using BRUV, $6 \%$ were Type I, 6\% were Type II, and $88 \%$ were Type III. Of the species observed only using UVC, $41 \%$ were Type I, $9 \%$ were Type II, and $50 \%$ were Type III.

Using UVC we recorded significantly more Type I and Type III species $\left(t_{(62)}=7.602\right.$ and $t_{(111)}=6.065$, respectively, both $\mathrm{p}<0.0005)$ and higher relative abundance of Type I species $\left(t_{(70)}=7.888, \mathrm{p}<0.0005\right)$ than using BRUV (Fig. 7). There was no difference between the methods in terms of relative abundance of Type II and Type III species $\left(t_{(73)}=-0.325\right.$ and $t_{(95)}=$ 1.211 , respectively) or in the number of Type II species $\left(t_{(57)}=1.747\right)($ Fig. 8) .

Using independent samples t-tests with the degrees of freedom computed using the Welch-Satterthwaite
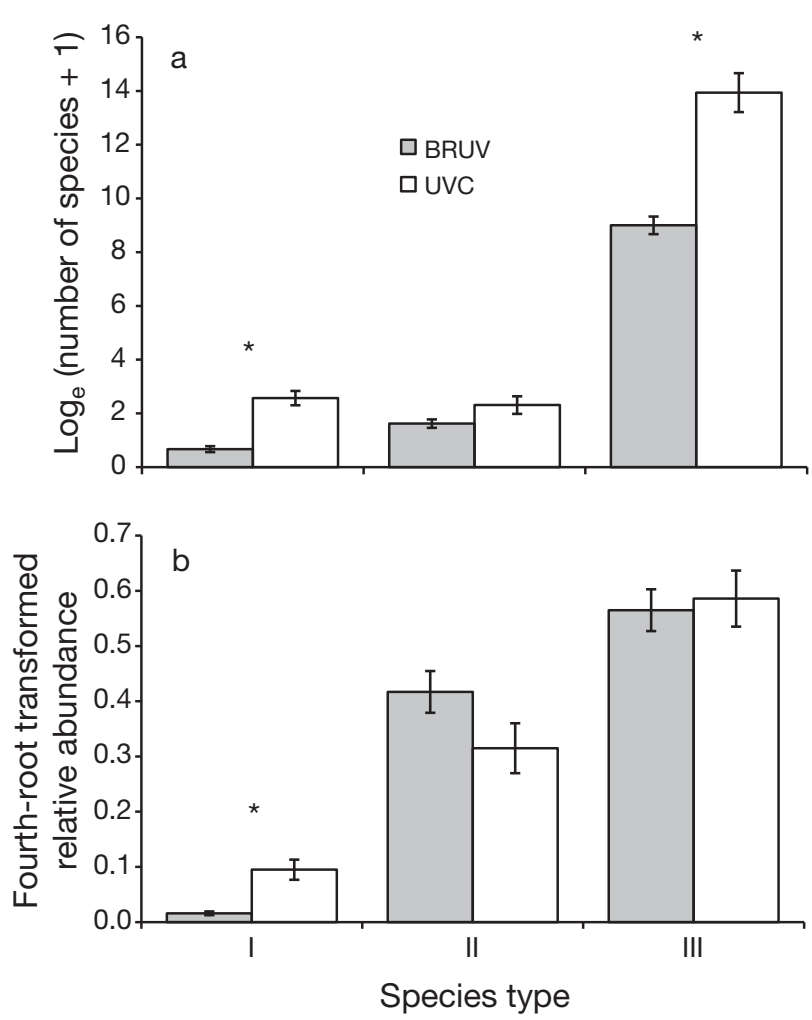

Fig. 7. Comparison between UVC and BRUV of sightability types (see 'Materials and Methods') for (a) numbers of species, and (b) relative numbers of individuals. Asterisks indicate significant differences at $p<0.0005$. Error bars are $\pm \mathrm{SE}$

formula, we found that UVC recorded significantly higher relative abundance of all herbivorous species (Fig. 8a), and 3 out of 4 territorial species (Fig. 8b). In comparison, BRUV observed significantly higher abundance of 7 of the 23 species of mobile predators (Fig. 8c, Table 3).

\section{Comparison of effort}

We constructed species accumulation curves using EstimateS (Colwell 2006) and then used Eq. (2) to compute $S_{\max }$ for each method at each location (Table 4). At all locations but Wilsons Promontory, $S_{\max }$ was higher using UVC than using BRUV. However, the differences in $S_{\max }$ were negligible at all locations except Cape Howe where predicted richness using UVC was 13 species higher than that predicted using BRUV. Using Eq. (3), we found that more UVC than BRUV samples were required to observe the same proportion of maximum species richness (Table 4), even without standardizing by the amount of time required to complete a sample of each method. When we standardized using 
the ratio of 2 UVCs completed in the time to deploy 1 BRUV, the difference in the number of samples required to observe a proportion of species richness grew. For example, at Barwon Heads, it would take 14 BRUV and 8 UVC samples to reach $60 \%$ of maximum species richness, and 180 BRUV and 106 UVC samples to observe $95 \%$ of predicted species richness.

\section{DISCUSSION}

This study represents one of the first assessments of how 2 methods, UVC and BRUV, compare in surveying subtidal fish assemblages. Unlike other comparisons between these methods (Willis \& Babcock 2000, Willis et al. 2000, Watson et al. 2005), we found that UVC
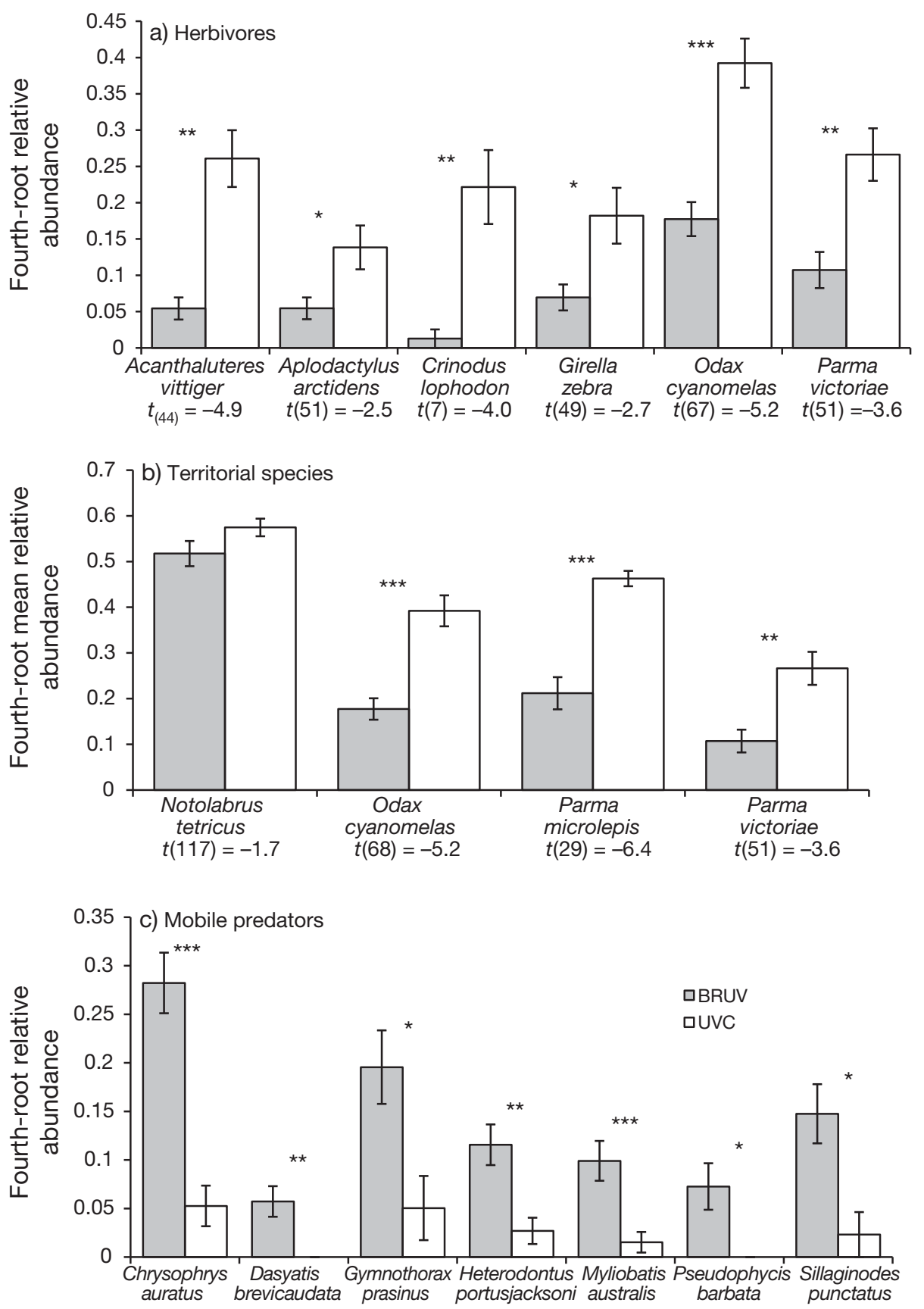

Fig. 8. Independent samples $t$-test results comparing mean relative abundance between methods for (a) herbivores, (b) territorial species, and (c) only those mobile predators for which a significant difference was found. (a) \& (b) results from t-tests given as $t_{\text {(df) }}$ below species' names; results for (c) are listed in Table 3. Asterisks indicate significance: ${ }^{*} \mathrm{p}<0.03{ }^{*}{ }^{* *} \mathrm{p}<0.001 ;{ }^{* * *} \mathrm{p}<$ 0.0005. Error bars are $\pm \mathrm{SE}$ 
Table 3. Independent samples t-tests results with df computed using the Satterthwatite-Welch formula, comparing between methods the fourth-root transformed mean relative abundance of mobile predators. Significance was assessed at $\alpha$-level $=0.03 ;$ significant $\mathrm{p}$-values and species are in bold

\begin{tabular}{|c|c|c|c|c|c|}
\hline Family & Species & $\bar{X}_{(\mathrm{BRUV})} \pm \mathrm{SE}$ & $\bar{X}_{(\mathrm{UVC})} \pm \mathrm{SE}$ & $t(\mathrm{df})$ & $\mathrm{p}$ \\
\hline Arripidae & Arripis spp. & $0.02 \pm 0.02$ & 0 & $1.0(21)$ & 0.329 \\
\hline Carangidae & Seriola lalandi & $0.03 \pm 0.02$ & 0 & $1.4(23)$ & 0.162 \\
\hline Congridae & Conger verreauxi & $0.02 \pm 0.02$ & 0 & $1.0(21)$ & 0.329 \\
\hline Dasyatidae & Dasyatis brevicaudata & $0.06 \pm 0.02$ & 0 & $3.6(84)$ & $<0.0005$ \\
\hline Dinolestidae & Dinolestes lewini & $0.15 \pm 0.03$ & $0.19 \pm 0.05$ & $-0.7(61)$ & 0.467 \\
\hline Heterodontidae & Heterodontus portusjacksoni & $0.12 \pm 0.02$ & $0.03 \pm 0.01$ & $3.6(118)$ & 0.001 \\
\hline Hexanchidae & Notorynchus cepedianus & $0.02 \pm 0.02$ & 0 & $1.0(21)$ & 0.329 \\
\hline Latridae & Latridopsis forsteri & $0.02 \pm 0.01$ & $0.09 \pm 0.04$ & $-1.7(32)$ & 0.092 \\
\hline Latridae & Latris lineata & $0.01 \pm 0.01$ & 0 & $1.0(21)$ & 0.329 \\
\hline Moridae & Pseudophycis barbata & $0.07 \pm 0.02$ & 0 & $3.0(44)$ & 0.004 \\
\hline Muraenidae & Gymnothorax prasinus & $0.20 \pm 0.04$ & $0.05 \pm 0.03$ & $2.9(24)$ & 0.008 \\
\hline Myliobatidae & Myliobatis australis & $0.10 \pm 0.02$ & $0.02 \pm 0.01$ & $3.6(114)$ & $<0.0005$ \\
\hline Orectolobidae & Orectolobus spp. & $0.01 \pm 0.01$ & $0.02 \pm 0.02$ & $-0.4(11)$ & 0.705 \\
\hline Platycephalidae & Platycephalus bassensis & $0.05 \pm 0.02$ & 0 & $2.0(44)$ & 0.050 \\
\hline Rajidae & Dipturus whitleyi & $0.02 \pm 0.01$ & 0 & $1.7(67)$ & 0.103 \\
\hline Rhinobatidae & Trygonorhina spp. & $0.10 \pm 0.02$ & $0.05 \pm 0.02$ & $1.6(77)$ & 0.120 \\
\hline Scyliorhinidae & Asymbolis spp. & $0.01 \pm 0.01$ & 0 & $1.0(23)$ & 0.328 \\
\hline Scyliorhinidae & Cephaloscyllium laticeps & $0.14 \pm 0.02$ & $0.07 \pm 0.02$ & $2.0(103)$ & 0.047 \\
\hline Sillaginidae & Sillaginodes punctatus & $0.15 \pm 0.03$ & $0.02 \pm 0.02$ & $3.3(84)$ & 0.002 \\
\hline Sparidae & Chrysophrys auratus & $0.28 \pm 0.03$ & $0.05 \pm 0.02$ & $6.1(117)$ & $<0.0005$ \\
\hline Sphyraenidae & Sphyraena novaehollandiae & $0.04 \pm 0.01$ & $0.01 \pm 0.01$ & $2.2(90)$ & 0.031 \\
\hline Triakidae & Mustelus antarcticus & $0.03 \pm 0.02$ & 0 & $1.4(23)$ & 0.170 \\
\hline Urolophidae & Various spp. & $0.02 \pm 0.01$ & $0.05 \pm 0.03$ & $-1.0(38)$ & 0.303 \\
\hline
\end{tabular}

Table 4. Predicted maximum species richness $\left(S_{\max }\right)$, determined from species accumulation curves, for each method at each location by fitting a Michaelis-Menten function (Eq. 2). Number of samples required by each method to achieve a proportion ( $Z$ ) of $S_{\max }$ (Eq. 3) is listed for each method at each location. Note these numbers have not been standardized by the relative amount of time required to complete a sample of each method

\begin{tabular}{|c|c|c|c|c|c|c|c|c|}
\hline \multirow[t]{3}{*}{ Location } & \multirow{2}{*}{\multicolumn{2}{|c|}{$S_{\max } \pm \mathrm{SE}$}} & \multicolumn{6}{|c|}{ Number of samples } \\
\hline & & & $\begin{array}{r}Z \\
\end{array}$ & $0 \%$ & & $\%$ & $\begin{array}{r}Z \\
\text { RRII }\end{array}$ & $\%$ \\
\hline & & & & & & & & \\
\hline Apollo & $46 \pm 0.6$ & $53 \pm 0.9$ & 7 & 4 & 14 & 8 & 90 & 53 \\
\hline Barwon & $44 \pm 0.8$ & $43 \pm 1.2$ & 6 & 4 & 13 & 9 & 82 & 56 \\
\hline Prom & $62 \pm 0.8$ & $56 \pm 1.8$ & 11 & 3 & 21 & 6 & 135 & 39 \\
\hline Howe & $58 \pm 0.2$ & $71 \pm 0.8$ & 6 & 2 & 13 & 5 & 82 & 31 \\
\hline
\end{tabular}

recorded more individuals and higher species diversity as measured by species richness, family richness and the Shannon Index (Fig. 4). At each location, we conducted up to 3 times more BRUV deployments than UVCs (Table 1). Based on these numbers alone, we would expect to record higher species diversity using BRUV than UVC. The fact that we found the opposite to be true suggests that these results may be conservative. Though we found UVC to record higher species richness, family richness, Shannon Index diversity and number of individuals, we determined that BRUV was the better method for observing mobile predators and species targeted by fishing. In addition, BRUV recorded a higher taxonomic diversity than UVC
(Fig. 6a), though this was not evident when the evenness of the taxonomic tree was taken into account (Fig. $6 \mathrm{~b})$. The higher taxonomic distinctness recorded using BRUV is most likely attributable to the higher species richness of elasmobranchs observed using this method. The difference between $\Delta^{+}$and $\Lambda^{+}$can be explained by the fact that the species that contributed to $\Delta^{+}$, e.g. Notorynchus cepedianus, were observed relatively infrequently using BRUV.

The differences between our results and those reported by other studies (Willis \& Babcock 2000, Willis et al. 2000, Watson et al. 2005) are likely to be, in part, a consequence of different approaches. Firstly, most of the previous studies examined the relative 
effectiveness of the methods for surveying only a few select species (Willis \& Babcock 2000, Willis et al. 2000). Only one study has compared the relative merits of BRUV and UVC as tools for surveying a fish community (Stobart et al. 2007). Using qualitative methods, Stobart et al. (2007) found that UVC recorded higher diversity and abundance of many species than BRUV, though these results were not statistically significant. The authors suggested that UVC was the better method especially considering its capacity to provide measures of absolute density.

The second and perhaps more important issue complicating comparisons is that of sampling area. For example, previous comparisons between UVC and BRUV have contrasted 30 min soak times with UVC transects of $125 \mathrm{~m}^{2}$ (Willis \& Babcock 2000, Willis et al. 2000). A BRUV unit would sample $314 \mathrm{~m}^{2}$ if the odour only dispersed $10 \mathrm{~m}$ from the bait in $30 \mathrm{~min}$ (Willis et al. 2000), which is most likely a significant underestimate (Willis et al. 2000, Harvey et al. 2007, Heagney et al. 2007). While models for estimating bait odour plume dispersal exist in deep waters (Sainte-Marie \& Hargrave 1987, Heagney et al. 2007), they have yet to be developed for turbulent environments leaving estimates of odour dispersal to speculation. However, like other authors (Willis et al. 2000), we feel it is safe to assume that a comparison between a $125 \mathrm{~m}^{2} \mathrm{UVC}$ and a 30 min BRUV deployment is a comparison between 2 different sampling areas; i.e. the BRUV is sampling a larger area than the UVC to which it is compared. The mean area surveyed in this study using UVC was $1790 \mathrm{~m}^{2}$, suggesting that our comparison may not be as compromised by differences in sample area as previous studies. However, as the area sampled by BRUV remains unknown, it is possible that our UVC surveys sampled a larger area than that sampled by the BRUV units, leading to the observed differences between methods.

Between-methods comparisons are inherently confounded by differences in the data collected. To standardize our measures of abundance, we conducted comparisons between relative abundance, i.e. proportions. Proportions are dependent upon the total number of individuals observed: The method that records more total individuals will record a lower proportion of individuals of a given species even if the same number of individuals is observed using both methods. If UVC had recorded fewer individuals in total, we would expect the proportion of a given species to be higher than that observed using BRUV. However, as this was not the case (Fig. 4), we are confident that those comparisons for which UVC observed higher relative abundance are valid. While the use of relative abundance will solve some of the complications arising from between-methods comparisons, issues remain. In par- ticular, the use of $\operatorname{Max} N$, which is a conservative measure, will by definition underestimate abundance. The use of $\operatorname{Max} N$ could therefore contribute to the lower abundance measured using BRUV.

It could be argued that we surveyed more habitat types using UVC than BRUV, resulting in higher species diversity for the method that encountered higher habitat diversity. However, the fact that we completed up to 3 times more BRUVs than UVC transects may serve to counter some of these concerns. Both types of surveys were conducted on rocky reefs, a patchy environment consisting of rocky outcrops separated by sand gullies, and both methods sampled similar habitats including the sand-reef interface and the reef itself. For differences in the number of habitats surveyed to fully explain our results requires that individuals be unable or unwilling to move between habitats in response to bait. While this may be true for some species, we did observe reef-associated species over sand on some videos, suggesting that BRUV may survey more than just the habitat into which it is deployed. However, we also found that UVC recorded higher relative abundance of 3 of 4 territorial species (Fig. 9b), suggesting that site fidelity may account for some of the differences between methods.

Several studies have found that divers are better than cameras at observing cryptic (Type I) species because divers are able to search complex habitats in ways that cameras cannot (Watson et al. 2005, Stobart et al. 2007). Using UVC, we observed more Type I species and more individuals of Type I species than using BRUV (Fig. 7a,b). Similarly, many of the species that were observed only using UVC were Type I, suggesting that at least some of the differences between methods can be explained by the better ability of divers to record Type I species. Overall, however, relatively few Type I species were recorded by either method, indicating that neither UVC nor BRUV is particularly effective at observing cryptic species. We estimate that only $38 \%$ of the species that could occur in the region were observed in our sampling (M. A. Colton unpubl. data). Like other studies (e.g. Willis 2001, Watson et al. 2005, Stobart et al. 2007), this underscores the need for additional types of survey methods, e.g. ichthyocides, if an adequate sampling of Type I species is desired.

The presence of Type II species could theoretically lead to the type of between-methods differences we observed. BRUV can underestimate density of these species because there is an upper limit to the number of fish that can be viewed in a frame (Willis et al. 2000). However, for only a few BRUV tapes at one location, Cape Howe, was the field of view completely filled with individuals of schooling species (e.g. Atypichthys strigatus, Caesioperca spp.). In contrast, UVC could overestimate density of these species through the 


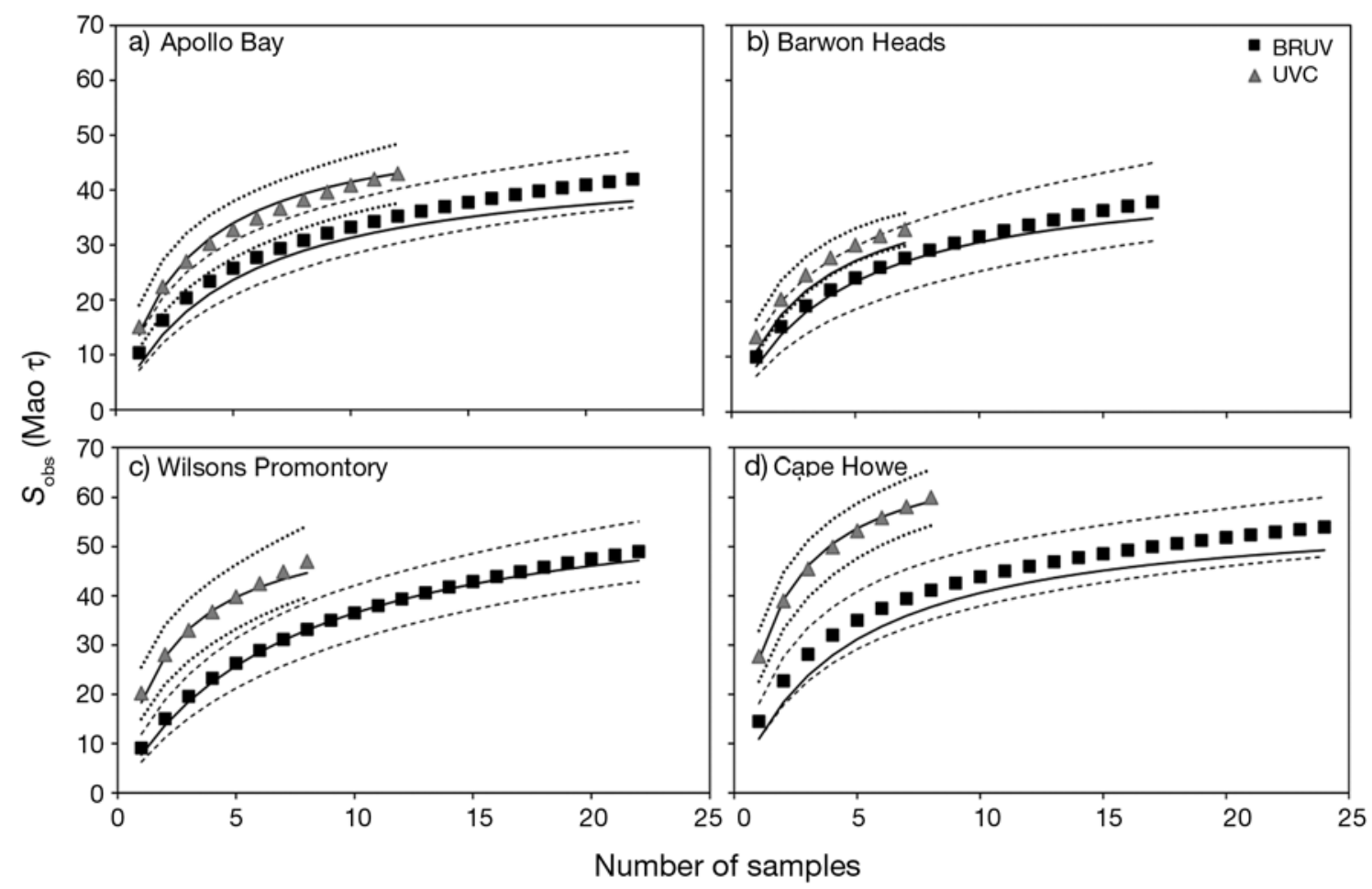

Fig. 9. Species accumulation curves for each method at each location. One BRUV sample = one BRUV deployment, and on average one UVC sample $\approx 360 \times 5 \mathrm{~m}$ strip transect. Dashed lines $=95 \%$ confidence intervals. Solid lines $=$ Michaelis-Menten functions described as (a) $y_{\text {(BRUV) }}=(46.2 x)(x+4.8)^{-1}$ and $y_{(\mathrm{UVC})}=(53.1 x)(x+2.8)^{-1}$; (b) $y_{\text {(BRUV) }}=(43.9 x)(x+4.3)^{-1}$ and $y_{\text {(UVC) }}=(43.5 x)$ $(x+3.0)^{-1} ;(\mathrm{c}) y_{(\mathrm{BRUV})}=(62.5 x)(x+7.1)^{-1}$ and $y_{(\mathrm{UVC})}=(56.2 x)(x+2.1)^{-1} ;(\mathrm{d}) y_{(\mathrm{BRUV})}=(58.1 x)(x+4.3)^{-1}$ and $y_{(\mathrm{UVC})}=(71.3 x)(x+1.6)^{-1}$. Predicted species richness $\left(S_{\max }\right)$ is listed for each method at each location

recounting of individuals. While we made every effort to avoid this, some individuals of schooling species that appeared to be attracted to divers (e.g. Scorpis spp. and Caesioperca spp.) were probably counted multiple times. However, only 2 Type II schooling species were identified by the SIMPER routine as contributing to the differences between methods: Caesioperca rasor and Scorpis aequipinnis (Table 2). As expected, both of these species were observed in higher numbers by UVC than BRUV. However, they contributed relatively little to the differences between methods: $3.1 \%$ and $2.8 \%$, respectively. Indeed, only $12 \%$ of all species observed were Type II species. In addition, an independent samples $t$-test found no difference between methods in the number or abundance of Type II species (Fig. 7), suggesting that densely schooling species cannot wholly account for differences between methods.

We found no significant difference between the methods in the relative abundance of Type II or III individuals (Fig. 7a). However, we found that UVC recorded significantly more Type III species than BRUV (Fig. 7a). As the majority of species observed were Type III (Fig. 3), and UVC observed more species than BRUV, it seems that the majority of differences between the 2 methods can be explained by differences in their ability to record Type III species. Indeed, of the species identified by SIMPER to explain differences between methods, the 2 contributing the most were both Type III, as were $75 \%$ of the species contributing to $50 \%$ of the between-method variation (Table 2).

One kind of Type III species for which there was a significant difference between methods was mobile predators. BRUV recorded higher mean relative abundance for 7 species of mobile predators, and UVC for none (Table 3). Some of the species better recorded by BRUV are important components of commercial fisheries, e.g. Chrysophrys auratus and Sillaginodes punctatus. At least 2 other studies (Willis \& Babcock 2000, Watson et al. 2005) also found that BRUV was better at observing targeted species. In addition, 5 species of elasmobranch were only observed using BRUV (Fig. 3), which is not surprising as diver-avoidance behaviour by these species has been documented elsewhere (e.g. Watson \& Harvey 2007). However, some researchers have found UVC to be an effective method with which to survey mobile predators (e.g. Friedlander \& DeMartini 2002, Castro \& Rosa 2005, Robbins et al. 2006). In using UVC to survey mobile predators, it 
may be important to sample larger width and length transects than were used in this research (e.g. Castro \& Rosa 2005, Robbins et al. 2006). However, Friedlander \& DeMartini (2002) surveyed smaller belt transects, both in terms of length and width, suggesting that the differences we observed between methods may not be wholly attributable to our UVC survey methodology. Indeed, in none of these studies were the densities measured using UVC compared to densities measured using BRUV. It is possible, as our results seem to suggest, that these studies underestimate the densities of elasmobranchs and that another method, such as BRUV, could have recorded higher densities.

Though Harvey et al. (2007) found that BRUV recorded herbivorous as well as carnivorous species, differences in species' attraction to bait could explain the differences between the methods. There are few data available about dietary preferences for the majority of species observed in this study; we were only able to identify 6 herbivores that were observed more than once during our surveys (Fig. 3). All 6 herbivores were better observed by UVC than BRUV (Fig. 8a), suggesting that dietary preference may explain at least part of the difference between these methods.

We also investigated whether territoriality could explain the differences between UVC and BRUV. For 3 of the 4 species identified as territorial, UVC observed more individuals than did BRUV (Fig. 8b). Two of these species are also herbivorous: the odacid Odax cyanomelas and the pomacentrid Parma victoriae. Their dietary ambivalence towards the bait combined with their territoriality may explain why more individuals of these species were observed by UVC than by BRUV. The 2 pomacentrids, $P$. microlepis and $P$. victoriae, which were better observed by UVC, are Type I species which were most often observed under ledges or at the entrance to caves. The inability of stationary cameras to observe these species in complex habitats offers an alternate explanation to between-methods differences. The single territorial species for which there was no significant difference in relative abundance was the labrid Notolabrus tetricus. This species is protogynous and haremic, with only the terminal phase displaying intra-specific antagonistic behaviour around the bait (M. A. Colton pers. obs.). Our inability to separate between phases in our data may mask a true between-methods difference in the relative abundance of this species. To definitively understand whether territoriality influences estimates of abundance recorded by these methods it will be necessary to repeat these comparisons in areas where more known territorial species occur.

In addition to investigating species-specific traits that cause differences between methods, we examined effort. At 2 locations, Wilsons Promontory and Cape
Howe, the $95 \%$ confidence intervals around species accumulation curves showed no overlap between BRUV and UVC, indicating that for a given number of non-standardized samples, UVC records more species than BRUV (Fig. 9). At the other 2 locations, Apollo Bay and Barwon Heads, the trend is the same. Based on the species accumulation curves, we computed $S_{\max }$ and found that at 3 locations $S_{\max }$ was higher for UVC than BRUV (Table 4). However, the species accumulation curves, and therefore $S_{\max }$ values, underestimate differences between methods by treating the samples of each method as equal. In our research, we computed that we could conduct twice as many UVCs as BRUVs in a given unit of time. When we standardized by time the number of samples required to achieve a proportion of $S_{\max }$ we found that many more BRUV than UVC samples were required. Therefore, in our research UVC more efficiently sampled species richness.

One of the biggest advantages in the use of BRUV is that units can be deployed in depths and locations that are inaccessible to SCUBA divers and at all times of day and night. Use of BRUV avoids the health and safety concerns associated with SCUBA and may allow more samples to be taken in a given unit of field time. Some research programs report that fewer personnel are required to launch and retrieve a BRUV unit as compared to conducting a UVC transect ( $\mathrm{T}$. Langlois pers. comm.), which may make BRUVs less costly than UVCs. Finally, BRUV provides a permanent record that can be repeatedly examined to ensure that fishes are correctly identified. However, there is a wellknown bottleneck in the processing of BRUV tapes (Willis et al. 2000, Cappo et al. 2003, Stobart et al. 2007). We computed that $\sim 2$ UVC transects can be conducted, including field and lab time, for every single BRUV unit deployed. It is important to note that this is probably an overestimate of the time to deploy a BRUV unit because we were working with only 2 frames; use of additional frames will reduce this time considerably. In our research, the ratio of time to deploy a BRUV vs. a UVC means that many more BRUVs than UVCs are required to record a certain percentage of predicted species richness, with the result that UVCs may cost less in terms of personnel hours than BRUVs.

\section{CONCLUSIONS}

Our comparison between 2 methods commonly used to survey subtidal fishes demonstrates that the method chosen to collect data will influence estimates of abundance. Using UVC, we obtained higher measures of species and family richness, diversity as measured by the Shannon Index, and recorded more individuals than using BRUV. We attribute the majority of these 
differences to the better ability of divers to search complex habitats as compared to a stationary camera. This is reflected in the significantly higher relative abundance and number of species of Type I recorded using UVC (Fig. 7a,b). In addition, it appears that BRUV underestimates the density of herbivorous and territorial species (Fig. 8a,b). In contrast, BRUV recorded higher species richness and abundance of mobile predators (Fig. 8c). Using BRUV we also recorded a more taxonomically diverse sample (Fig. 6a), though this difference was eliminated when taxonomic evenness was taken into account (Fig. 6b). These results suggest that studies wishing to catalogue diversity would do best to use a variety of methods, whereas those which target only a few select species would do best to select the method and size of sampling area which best suits those species. We are not alone in these recommendations: Many other studies have suggested that a combination of techniques may be necessary to survey an entire fish assemblage (e.g. Sale \& Douglas 1981, Sale \& Sharp 1983, Lincoln Smith 1988). However, in situations in which financial or time constraints limit researchers to only a single method, our results suggest that UVC will likely be the better option.

Acknowledgements. This research was funded by Natural Heritage Trust and Parks Victoria. Field assistance was provided by D. Chamberlain, J. Ford, M. LeFeuvre, M. Lindsay, C. Jung, and Parks Victoria staff. M. Gomon and D. Bray assisted with difficult fish identifications, and 4 anonymous reviewers provided valuable feedback on earlier versions of this manuscript.

\section{LITERATURE CITED}

Boulinier T, Nichols JD, Sauer JR, Hines JE, Pollock KH (1998) Estimating species richness: the importance of heterogeneity in species detectability. Ecology 79:1018-1028

Brock VE (1954) A preliminary report on a method of estimating reef fish populations. J Wildl Manag 18:297-308

Cappo M (2006) Illustrated guide to assembly, deployment and retrieval of BRUVS. Australian Institute of Marine Science, Townsville

Cappo M, Harvey E, Malcolm H, Speare P (2003) Potential of video techniques to monitor diversity, abundance and size of fish in studies of marine protected areas. In: Beuner JP, Grant A, Smith DC, Mahon D (eds) Aquatic Protected Areas: What works best and how do we know? Proceedings of the World Congress on Aquatic Protected Areas, Cairns, August 2002, p 455-464

Cappo M, Speare P, De'ath G (2004) Comparison of baited remote underwater video stations (BRUVS) and prawn (shrimp) trawls for assessments of fish biodiversity in interreefal areas of the Great Barrier Reef Marine Park. J Exp Mar Biol Ecol 302:123-152

Cappo M, Harvey E, Shortis M (2007) Counting and measuring fish with baited video techniques - an overview. In: Lyle JM, Furlani DM, Buxton CD (eds) Cutting-edge technologies in fish and fisheries science. Australian Society for Fish Biology Workshop Proceedings, Hobart, August
2006, p 101-114

Castro ALF, Rosa RS (2005) Use of natural marks on population estimates of the nurse shark, Ginglymostoma cirratum, at Atol das Rocas Biological Reserve, Brazil. Environ Biol Fishes 72:213-221

Clarke KR, Warwick RM (1998) A taxonomic distinctness index and its statistical properties. J Appl Ecol 35:523-531

Clarke KR, Warwick RM (2001a) Change in marine communities: an approach to statistical analysis and interpretation. Primer-E Ltd, Plymouth

> Clarke KR, Warwick RM (2001b) A further biodiversity index applicable to species lists: variation in taxonomic distinctness. Mar Ecol Prog Ser 216:265-278

Colwell RK (2006) EstimateS: statistical estimation of species richness and shared species from samples. Version 8.0. User's guide and application available at: http://viceroy. eeb.uconn.edu/EstimateS

Edgar GJ (2005) Australian marine life: the plants and animals of temperate waters. Reed New Holland, Sydney

- Edgar GJ, Barrett NS, Morton AJ (2004) Biases associated with the use of underwater visual census techniques to quantify the density and size-structure of fish populations. J Exp Mar Biol Ecol 308:269-290

Eschmeyer WN, Fricke R (2009) Catalog of Fishes electronic version, available at: http://research.calacademy.org/ ichthyology/catalog/fishcatmain.asp (accessed 9 September 2009)

Farnsworth KD, Thygesen UH, Ditlevsen S, King NJ (2007) How to estimate scavenger fish abundance using baited camera data. Mar Ecol Prog Ser 350:223-234

> Fowler AJ (1987) The development of sampling strategies for population studies of coral reef fishes: a case study. Coral Reefs 6:49-58

> Friedlander AM, DeMartini EE (2002) Contrasts in density, size, and biomass of reef fishes between the northwestern and the main Hawaiian islands: the effects of fishing down apex predators. Mar Ecol Prog Ser 230:253-264

Gomon MF, Bray D, Kuiter R (eds) (2008) Fishes of Australia's southern coast. Reed New Holland, Sydney

> Harvey E, Fletcher D, Shortis MR, Kendrick GA (2004) A comparison of underwater visual distance estimates made by scuba divers and a stereo-video system: implications for underwater visual census of reef fish abundance. Mar Freshw Res 55:573-580

> Harvey ES, Cappo M, Butler JJ, Hall N, Kendrick GA (2007) Bait attraction affects the performance of remote underwater video stations in assessment of demersal fish community structure. Mar Ecol Prog Ser 350:245-254

Heagney EC, Lynch TP, Babcock RC, Suthers IM (2007) Pelagic fish assemblages assessed using mid-water baited video: standardising fish counts using bait plume size. Mar Ecol Prog Ser 350:255-266

Hough D, Mahon G (1994) Biophysical classification of Victoria's marine waters. In: Muldoon J (ed) Towards a marine regionalisation for Australia. Great Barrier Reef Marine Park Authority, Sydney

Kuiter RH (2000) Coastal fishes of South-eastern Australia. Gary Allen, Sydney

Kulbicki M (1998) How the acquired behaviour of commercial reef fishes may influence the results obtained from visual censuses. J Exp Mar Biol Ecol 222:11-30

- Lincoln Smith MP (1988) Effects of observer swimming speed on sample counts of temperate rocky reef fish assemblages. Mar Ecol Prog Ser 43:223-231

Lincoln Smith MP (1989) Improving multispecies rocky reef fish censuses by counting different groups of species using different procedures. Environ Biol Fishes 26:29-37 
MacNeil MA, Graham NAJ, Conroy MJ, Fonnesbeck CJ, Polunin NVC, Rushton SP, Chabanet P, McClanahan TR (2008a) Detection heterogeneity in underwater visualcensus data. J Fish Biol 73:1748-1763

MacNeil MA, Tyler EHM, Fonnesbeck CJ, Rushton SP, Polunin NVC, Conroy MJ (2008b) Accounting for detectability in reef-fish biodiversity estimates. Mar Ecol Prog Ser 367:249-260

Miller RJ (1975) Density of commercial spider crab, Chionoecetes opilio, and calibration of effective area fished per trap using bottom photography. J Fish Res Board Can 32: 761-768

Patterson HM, Lindsay M, Swearer SE (2007) Use of sonar transects to improve efficiency and reduce potential bias in visual surveys of reef fishes. Environ Biol Fishes 78:291-297

> Powter DM, Gladstone W (2008) The reproductive biology and ecology of the Port Jackson shark Heterodontus portusjacksoni in the coastal waters of eastern Australia. J Fish Biol 72:2615-2633

Priede IG, Merrett NR (1996) Estimation of abundance of abyssal demersal fishes; a comparison of data from trawls and baited camera. J Fish Biol 49:207-216

Raaijmakers JGW (1987) Statistical analysis of the MichaelisMenten equation. Biometrics 43:793-803

Robbins WD, Hisano M, Connolly SR, Choat JH (2006) Ongoing collapse of coral-reef shark populations. Curr Biol 16: 2314-2319

Sainte-Marie B, Hargrave BT (1987) Estimation of scavenger abundance and distance of attraction to bait. Mar Biol 94: 431-443

Sale PF, Douglas WA (1981) Precision and accuracy of visual census technique for fish assemblages on coral patch reefs. Environ Biol Fishes 6:333-339

Editorial responsibility: John Choat,

Townsville, Australia
Sale PF, Sharp BJ (1983) Correction for bias in visual transect censuses of coral reef fishes. Coral Reefs 2:37-42

Samoilys MA, Carlos G (2000) Determining methods of underwater visual census for estimating the abundance of coral reef fishes. Environ Biol Fishes 57:289-304

Sherrod PH (2008) Nonlinear regression analysis program (NLREG), Version 6.4. Nashville, TN

Stobart B, Garcia-Charton JA, Espejo C, Rochel E, Goni R, Renones O, Herrero A, Crec'hriou R, Polti S, Marcos C, Planes S, Perez-Ruzafa A (2007) A baited underwater video technique to assess shallow-water Mediterranean fish assemblages: Methodological evaluation. J Exp Mar Biol Ecol 345:158-174

Watson DL, Harvey ES (2007) Behaviour of temperate and sub-tropical reef fishes towards a stationary SCUBA diver. Mar Freshwat Behav Physiol 40:85-103

Watson DL, Harvey ES, Anderson MJ, Kendrick GA (2005) A comparison of temperate reef fish assemblages recorded by three underwater stereo-video techniques. Mar Biol 148:415-425

Willis TJ (2001) Visual census methods underestimate density and diversity of cryptic reef fishes. J Fish Biol 59:1408-1411

Willis TJ, Babcock RC (2000) A baited underwater video system for the determination of relative density of carnivorous reef fish. Mar Freshw Res 51:755-763

Willis TJ, Millar RB, Babcock RC (2000) Detection of spatial variability in relative density of fishes: comparison of visual census, angling, and baited underwater video. Mar Ecol Prog Ser 198:249-260

- Yau C, Collins MA, Bagley PM, Everson I, Nolan CP, Priede IG (2001) Estimating the abundance of Patagonian toothfish Dissostichus eleginoides using baited cameras: a preliminary study. Fish Res 51:403-412

Submitted: July 1, 2009; Accepted: October 19, 2009 Proofs received from author(s): January 27, 2010 